\title{
Acceleration of Brain TOF-MRA with Compressed Sensitivity Encoding: A Multicenter Clinical Study
}

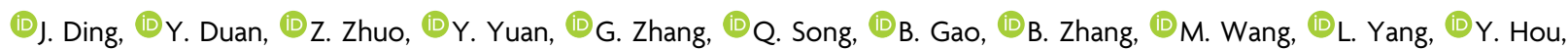
(D). Yuan, (1) C. Feng, ㄱ. J. Wang, (1) L. Lin, and (1)Y. Liu

\begin{abstract}
BACKGROUND AND PURPOSE: The clinical practice of three-dimensional TOF-MRA, despite its capability in brain artery assessment, has been hampered by the relatively long scan time, while recent developments in fast imaging techniques with random undersampling has shed light on an improved balance between image quality and imaging speed. Our aim was to evaluate the effectiveness of TOF-MRA accelerated by compressed sensitivity encoding and to identify the optimal acceleration factors for routine clinical use.
\end{abstract}

MATERIALS AND METHODS: One hundred subjects, enrolled at 5 centers, underwent 8 brain TOF-MRA sequences: 5 sequences using compressed sensitivity encoding with acceleration factors of 2, 4, 6, 8, and 10 (CS2, CS4, CS6, CS8, and CS10), 2 using sensitivity encoding with factors of 2 and 4 (SF2 and SF4), and 1 without acceleration as a reference sequence (RS). Five large arteries, 6 medium arteries, and 6 small arteries were evaluated quantitatively (reconstructed signal intensity, structural similarity, contrast ratio) and qualitatively (scores on arteries, artifacts, overall image quality, and diagnostic confidence for aneurysm and stenosis). Comparisons were performed among the 8 sequences.

RESULTS: The quantitative measurements showed that the reconstructed signal intensities of the assessed arteries and the structural similarity consistently decreased as the compressed sensitivity encoding acceleration factor increased, and no significant difference was found for the contrast ratios in pair-wise comparisons among SF2, CS2, and CS4. Qualitative evaluations showed no significant difference in pair-wise comparisons among RS, SF2, and CS2 (P>.05). The visualization of all the assessed arteries was acceptable for CS2 and CS4, while 2 small arteries in images of CS6 were not reliably displayed, and the visualization of large arteries was acceptable in images of CS8 and CS10.

CONCLUSIONS: CS4 is recommended for routine brain TOF-MRA with balanced image quality and acquisition time; CS6, for examinations when small arteries are not evaluated; and CS10, for fast visualization of large arteries.

ABBREVIATIONS: $\mathrm{ACA}=$ anterior cerebral artery; $\mathrm{BA}=$ basilar artery; $\mathrm{CR}=$ contrast ratio; $\mathrm{CS}=$ compressed sensing; $\mathrm{L}=$ left; $\mathrm{R}=$ right; $\mathrm{RS}=$ reference sequence without SENSE or CS-SENSE acceleration; RSI = reconstructed signal intensity; SENSE = sensitivity encoding; SF = SENSE technique with acceleration factors; SSIM = structural similarity index; VA = vertebral artery; PCA = posterior cerebral artery

$\mathbf{T}$ OF-MRA is a noninvasive routine clinical method that does not require intravenous contrast agents or exposure to radiation for brain artery assessment. ${ }^{1-3}$ Previous studies have

Received October 25, 2020; accepted after revision January 10, 2021.

From the Department of Radiology (J.D., Y.D., Z.Z., J.Y., .C.F., Y.L.), Beijing Tiantan Hospital, Capital Medical University, Beijing, China; Department of Radiology (Y.Y. G.Z.), Beijing Royal Integrative Medicine Hospital, Beijing, China; Department of Radiology (Q.S., B.G.), the First Affiliated Hospital of Dalian Medical University, Dalian, China; Department of Radiology (B.Z., M.W.), The Affiliated Drum Tower Hospital of Nanjing University Medical School, Jiangsu, China; Department of Radiology (L.Y., Y.H.), Shengjing Hospital of China Medical University, Shenyang, China; and Philips Healthcare (J.W., L.L.), Beijing, China.

This work was supported by the National Science Foundation of China (Nos. 81870958 and 81571631 ), the Beijing Natural Science fund (No.7133244), and the Beijing Nova Program (xx2013045).

Paper previously presented, in part, at: Annual Meeting of the International Society for Magnetic Resonance in Medicine and the Society for MR Radiographers and Technologists, August 8-14, 2020; Virtual. demonstrated its high diagnostic efficacy in detecting cerebrovascular diseases, which is comparable with that of DSA, and TOFMRA has been used to replace DSA in the diagnosis of cerebrovascular diseases for a range of clinical practices. ${ }^{2-5}$ However, the relatively long scan time of conventional TOF-MRA can potentially result in an uncomfortable experience for patients and increased motion artifacts in images. ${ }^{6-8}$ Parallel imaging techniques such as sensitivity encoding (SENSE) and generalized

Please address correspondence to Yaou Liu, MD, PhD, Department of Radiology, Beijing Tiantan Hospital, Capital Medical University. No.119, West Southern 4th Ring Rd, Fengtai District, Beijing, 100070, China; e-mail: yaouliu80@163.com

\section{- Indicates open access to non-subscribers at www.ajnr.org}

Indicates article with online supplemental data.

http://dx.doi.org/10.3174/ajnr.A7091 
autocalibrating partially parallel acquisition have been implemented in clinical practice in the past 2 decades for MR imaging acceleration. ${ }^{9,10}$ However, the acceleration capability of parallel imaging is limited by the number of receive coils, and the acceleration factor rarely goes beyond 4 in clinical setups due to potential imaging artifacts and signal-to-noise ratio concerns. ${ }^{10,11}$

The compressed sensing (CS) technique by pseudorandom $k$ space undersampling has shown its potential in fast MR imaging with a relatively high acceleration factor and suppressed imaging artifacts. $^{6,12-15}$ Previous studies have shown that CS-accelerated TOF-MRA outperforms traditional parallel imaging through quantitative image evaluation, and the CS-accelerated sequence has been applied for evaluating cerebral artery malformations, aneurysms, and stenoses. ${ }^{6,16-18}$ In previous studies of CS TOFMRA, most cerebral aneurysms were recognized using a CS factor of 5 or 8 with 10 iterations. $^{6}$ The diagnostic quality of several distal arterial branches could be maintained on images with a CS factor of up to $6^{12}$ and improved visualization of small collaterals in equivalent time (factor of 3); or equivalent results with a shorter scan time (factor of 5) could be achieved by CS in comparison with parallel imaging. ${ }^{18}$ However, most of these studies were performed at a single center or with a small number of subjects, and there has been no consensus on the optimal CS acceleration factor for 3D TOF-MRA. Therefore, a more comprehensive study is required to verify the effectiveness of CS on TOF-MRA and to identify the optimal CS acceleration factors for routine clinical use, preferably in a multicenter study with more subjects.

In this work, we aimed to evaluate the effectiveness of CSaccelerated TOF-MRA and identify the optimal CS acceleration factors for clinical use in a cohort of 100 subjects from 5 centers by systematically evaluating the image quality and diagnostic efficacy of CS-accelerated TOF-MRA with 5 different acceleration factors ranging from 2 to 10 . These findings were compared with results obtained by conventional SENSE with 2 acceleration factors and a nonaccelerated sequence.

\section{MATERIALS AND METHODS \\ Ethics}

This multicenter study was approved by the relevant institutional review boards. Informed consent was obtained from all participants.

\section{Study Population}

Between March 2019 and January 2020, we prospectively and consecutively enrolled participants at 5 different hospitals (located in different cities in China): center 1: Beijing Tiantan Hospital, Capital Medical University, in Beijing, 26 cases; center 2: Beijing Royal Integrative Medicine Hospital, in Beijing, 30 cases; center 3: The Affiliated Drum Tower Hospital of Nanjing University Medical School, in Nanjing, 23 cases; center 4: the First Affiliated Hospital of Dalian Medical University, in Dalian, 15 cases; and center 5: Shenging Hospital of China Medical University, in Shenyang, 14 cases. The inclusion criteria were as follows: 1) older than 18 years of age, and 2) healthy volunteers or participants suspected or confirmed of having cerebrovascular diseases. The exclusion criteria are included in Fig 1.

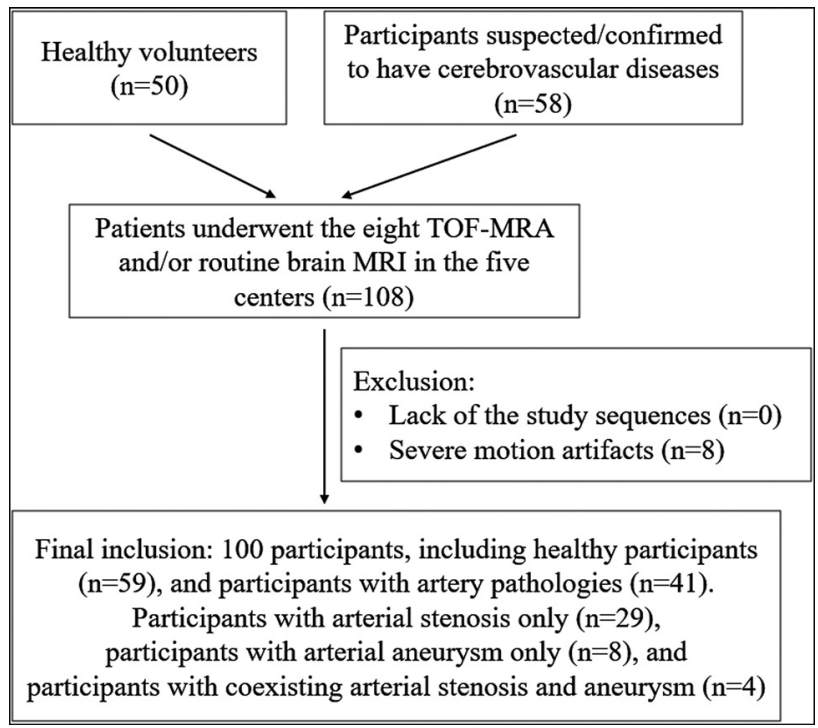

FIG 1. Flow chart for participant inclusion.

\section{MR Imaging Protocols}

Each participant was scanned head-first in the supine position at 3T (Ingenia CX; Philips Healthcare) at 1 of the 5 centers where the MR imaging systems were located, with a 32-channel head coil using compressed SENSE as the acceleration technique ${ }^{19,20}$ (a combination of CS and SENSE, hereafter referred to as CSSENSE) with the reconstruction algorithm shown in Equation 1, which essentially followed the technique described by Lustig and Pauley: ${ }^{21}$

$$
\begin{aligned}
& p=\min \left(\sum_{i=1}^{\sharp c o i l s}\left\|m_{d, i}-E S_{d, i} p\right\|_{2}^{2}+\lambda_{1}\left\|R^{-1 \backslash 2} p\right\|_{2}^{2}+\lambda_{2}\right. \\
& \left.\|\Psi p\|_{1}\right)
\end{aligned}
$$

where $p$ is the image to be reconstructed; $m_{d, i}$ is the measured value for a given coil element after noise decorrelation; $E$ is the undersampled Fourier operator defined by the subsampling pattern; $S_{d, i}$ is the coil sensitivity for a given coil element after noise decorrelation (obtained with the SENSE reference scan); $\lambda_{1}$ is the regularization factor for balancing between data consistency and prior knowledge of image content; $R$ is coarse resolution data from the integrated body coil obtained with the SENSE reference scan (used to constrain the solution during the regularization process); $\lambda_{2}$ is the regularization factor to balance the sparsity constrain and data consistency in the iterative solution; and $\Psi$ is the sparsity transform into the wavelet domain.

Each participant underwent 8 customized 3D TOF-MRA protocols (predesigned and optimized at Beijing Tiantan Hospital and then replicated on the MR imaging systems of other hospitals) in a random order. First, a routine brain MR imaging, including transverse T2-weighted TSE, sagittal 3D T1 turbo-field echo (known as MPRAGE), sagittal 3D-FLAIR, DWI, and SWI, was performed for all participants with suspected or confirmed cerebrovascular diseases, and after a period of 5-10 minutes, the 8 TOF-MRA sequences were additionally scanned. For healthy volunteers, routine brain MR imaging was optional, but the 8 TOF-MRA images were required. Among the 8 protocols, 5 used 
CS-SENSE with acceleration factors of 2, 4, 6, 8, and 10 (denoted as CS2, CS4, CS6, CS8, and CS10, respectively); 2 used the conventional SENSE technique with acceleration factors of 2 and 4 (denoted as SF2 and SF4, respectively); and 1 was a reference protocol without SENSE or CS-SENSE acceleration (denoted as RS). A $3 \mathrm{D}$ FOV was used to cover most cerebral arteries and the pericallosal artery branches, including the intracranial segment of the bilateral ICAs, the anterior cerebral artery (ACA, A1-A3 segments), the MCA (M1-M4 segments), the distal bilateral vertebral arteries (VAs, at least half of the V4 segment), the basilar artery (BA), and the posterior cerebral artery (PCA, P1-P3 segments). A saturation band was placed above the FOV to suppress the signals from veins. The parameters for the $8 \mathrm{MRA}$ protocols are listed in the Online Supplemental Data. The reconstruction was performed in real-time during the scan and did not affect the workflow of the hospital.

\section{Image Evaluation}

Images were transferred to the IntelliSpace Portal, Version 7.0 (Philips Healthcare) workstation and processed before evaluations. First, all images were checked visually to exclude data with insufficient quality due to unacceptable motion artifacts. Second, image realignment was performed using SPM 12 (www.fil.ion. ucl.ac.uk/spm/) for images acquired by the 8 sequences on the same subject. Third, the patient and sequence information was removed from all images. Finally, quantitative and qualitative assessments were performed.

Quantitative image evaluations were performed on Matlab R2016b (MathWorks). Because the iterative reconstruction of CSSENSE can result in artificial reduction of noise in MR images, it would be inaccurate to use classic measurement approaches for calculating the signal-to-noise ratio and/or contrast-to-noise ratio. Instead, the reconstructed signal intensities (RSIs) of 11 cerebral arteries, WM, and CSF were extracted from the source image-based ROIs drawn by a neuroradiologist (Y.D., with $>10$ years of experience). For the signal intensity measurements of the arteries, WM, and CSF for each subject, ROIs were placed on the same slices of the 8 TOF-MRA source images. ROIs of the ACA, MCA, or PCA (such as the $\mathrm{A} 1, \mathrm{M} 1$, and $\mathrm{P} 1$ segments) were drawn in the proximal segment, while ROIs of the ICA and VA (such as C7 and V4) were drawn in the distal segment. In the case of stenosis, slight anatomic variation, or occlusion, the proximal or distal segment with maximal display in the same section of the 8 TOF-MRA source images was selected for ROI measurements. The signal of the corpus callosum was used to represent the WM signal. ${ }^{22}$ The CSF signal was mainly acquired from the lateral ventricle. The contrast ratios (CRs) between assessed arteries and WM $\left(C R_{\text {artery/wm }}\right)$ and between assessed arteries and $\operatorname{CSF}\left(C R_{\text {artery/csf }}\right)$ were calculated in Equation 2 as $^{23}$

$$
C R_{\text {tissue } 1 / \text { tissue } 2}=\frac{\left|\mu_{\text {tissue } 1}-\mu_{\text {tissue } 2}\right|}{\sqrt{\sigma_{\text {tissue } 1}^{2}+\sigma_{\text {tissue } 2}^{2}}}
$$

where $\mu_{\text {tissue } 1}$ and $\mu_{\text {tissue } 2}$ are the ROI-based mean signal intensities of 2 specific tissues, with $\sigma_{\text {tissue } 1}$ and $\sigma_{\text {tissue } 2}$ as the corresponding variances. The assessed cerebral arteries included the left and right ICAs (LICA, RICA), ACAs (LACA, RACA), MCAs (LMCA, RMCA), VAs (LVA, RVA), PCAs (LPCA and RPCA), and the BA. In addition, the structural similarity index $(\mathrm{SSIM})^{24}$ was calculated to measure the image similarity between each of the 7 accelerated scans and the RS scan. ${ }^{20}$

The whole MIP radial axis in the foot-head direction (radial angle $=12^{\circ}$, fifteen projections in total, no preprocessing) was used for visual depiction of the cerebral arteries. Qualitative image evaluation was performed independently by 2 neuroradiologists (J.Y. and C.F., with $>8$ years of experience who were blinded to the patient information and imaging parameters). The image quality; visualization of large, medium, and small arteries; and diagnostic confidence of arterial pathologies for all 8 protocols were assessed according to the scoring system listed in the Online Supplemental Data. ${ }^{15}$ Before the evaluation, the 2 neuroradiologists completed a training session with 5 patients on the evaluation of the imaging findings. ${ }^{14}$ The mean scores of the 2 readers were used for subsequent statistical analyses.

Nonvisible distal arteries or branches due to severe stenosis or occlusion were excluded from further analyses, but the normal arteries in the same patient were still included. The criteria were as follows: protocols with mean scores for large and medium arteries of $\geq 3$, small arteries of $\geq 2$, artifacts of $\geq 2$, overall image quality of $\geq 3$, and diagnostic confidence of $\geq 2$ were considered acceptable for clinical setup.

\section{Statistical Analysis}

Statistical analyses were performed using Matlab R2016b (MathWorks). Repeated measures ANOVA tests were performed for the RSIs, CRs, and SSIM among the 8 sequences, while differences between each pair of protocols were evaluated by multiple comparisons with $P$ values corrected by the Bonferroni correction. The interobserver reliability on qualitative evaluation was assessed through the Cohen $\kappa$ test (excellent agreement if $\kappa>0.9$, good agreement if $\kappa>0.6$ ). Qualitative scores from all sequences were tested using the Friedman test, and multiple comparisons between each pair of the 8 sequences were performed with $P$ values corrected by the Bonferroni correction. For all tests, $P<.05$ was considered statistically significant.

\section{RESULTS}

\section{Participant Cohort}

A total of 108 participants from the 5 centers were initially recruited. Data from 8 participants were excluded due to motion artifacts. Finally, 100 participants (46 men and 54 women; mean age, 52.9 [SD, 16.5] years; age range, 23-88 years) were enrolled (Fig 1). Fifty-nine participants had no artery pathologies and included 50 healthy volunteers and 9 participants from the suspected group. Forty-one participants showed artery pathologies, including arterial stenosis only $(n=29)$, arterial aneurysm only $(n=8)$, or coexisting arterial stenosis and an aneurysm $(n=4)$. The locations of the arterial stenoses and aneurysms are listed in the Online Supplemental Data.

\section{Quantitative Measurements}

RSIs and SSIM. For all assessed arteries, WM, and CSF, significant differences were observed for RSIs among the 8 protocols $(P<.05)$, as well as the SSIM among the 7 accelerated protocols $(P<.05)$. For each artery, WM, or CSF, as the SENSE or CSSENSE acceleration factor increased, the mean reconstructed signal 


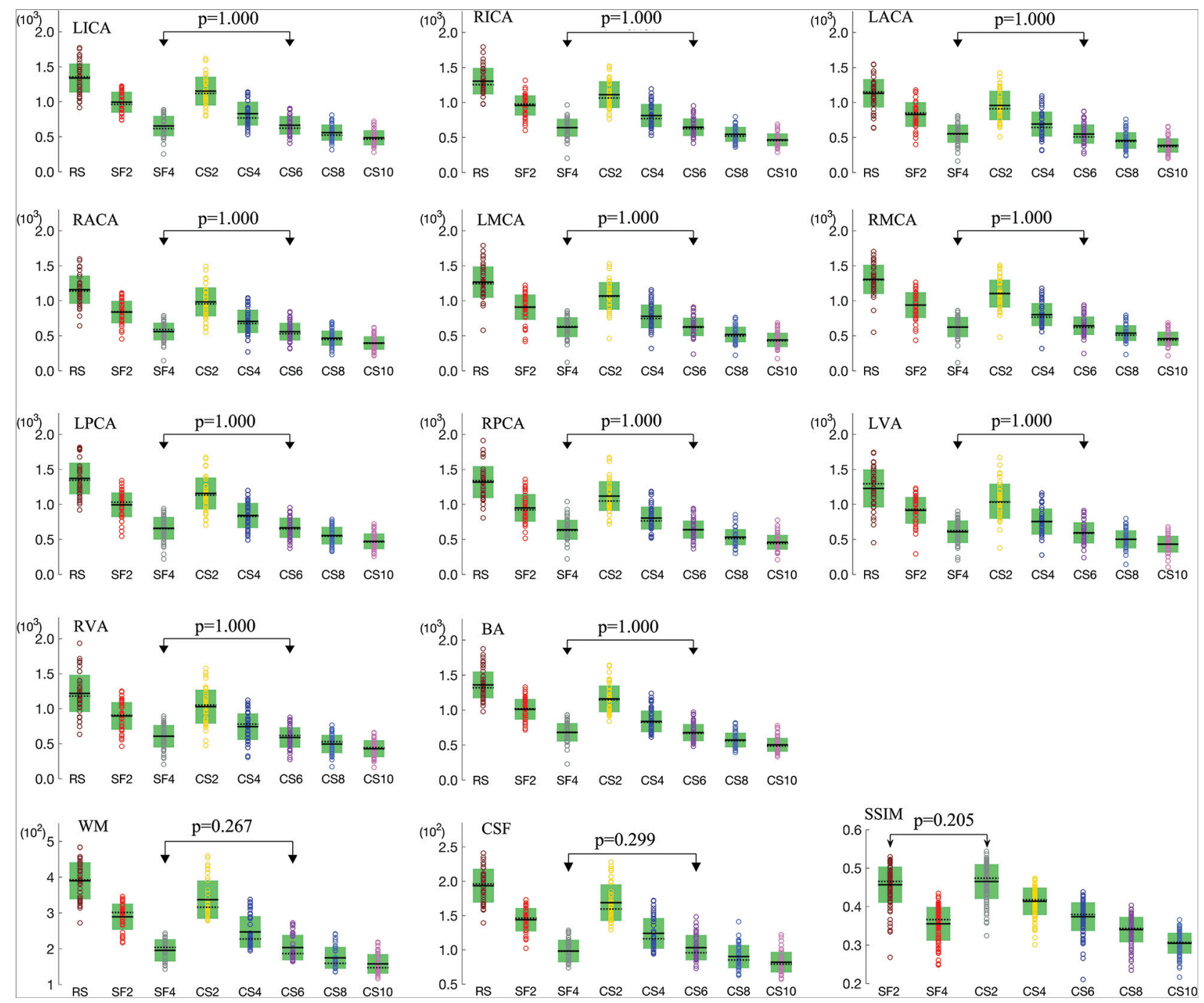

FIG 2. Measured RSIs for 11 arteries (LICA, RICA, LACA, RACA, LMCA, RMCA, LPCA, RPCA, LVA, RVA, and BA), WM, and CSF by the 8 sequences; and the SSIM values for the 7 accelerated scans with reference to the RS scan (circles indicate individual measurements; black lines, mean values; dotted black lines, median values; and green boxes, SD). Pairs without significant differences $(P>.05)$ are connected with double arrow lines because the $P$ values in other pairs were all .000.

intensity gradually decreased, and significant differences were found between any 2 of the 8 protocols $(P<.05$, Fig 2) except between SF4 and CS6. The SSIM also decreased as the SENSE or CS-SENSE acceleration factor increased, and no significant difference was found between the SF2 and CS2 scans $(P>.05$, Fig 2$)$.

Contrast Ratio. The mean $C R_{\text {artery/wm }}$ and $C R_{\text {artery/csf }}$ values decreased slightly and gradually for most arteries when the CSSENSE acceleration factor increased (Online Supplemental Data). Differences were found among the $8 \mathrm{MRA}$ sequences for the CRs using repeated measures ANOVA. Comparisons between any 2 of the 8 sequences are listed in the Online Supplemental Data. No significant difference was found for the CRs between any pairs of SF2, CS2, and CS4.

\section{Qualitative Assessments}

Good or excellent agreement was reached between the 2 readers for scoring artery visualization $(\kappa \geq 0.812)$, artifacts $(\kappa \geq 0.922)$, overall image quality $(\kappa \geq 0.901)$, and diagnostic confidence $(\kappa$ $\geq 0.894$ ).

Visualization of the Assessed Arteries. Significant differences were found for the scores of all arteries among the 8 protocols $(P<.05$, Online Supplemental Data). No significant difference was found in pair-wise comparison between RS, SF2, and CS2 for all arteries $(P>.05$, Online Supplemental Data). Images of RS, SF2, CS2, and CS4 were acceptable for the assessment of all arteries. Visualization of 2 small arteries (left anterior choroidal artery $[\mathrm{LAChA}]$ and right anterior choroidal artery [RAChA]) became less reliable (mean score $<2$, Online Supplemental Data) in CS6 images. Reliable visualization of large arteries was still maintained on images of CS8 and CS10 (mean scores $>3$ ) but not for some medium-sized and all the small-sized arteries.

Artifacts and Overall Image Quality. A typical artifact generated by SENSE was observed in SF4, and speckled noise was found in 

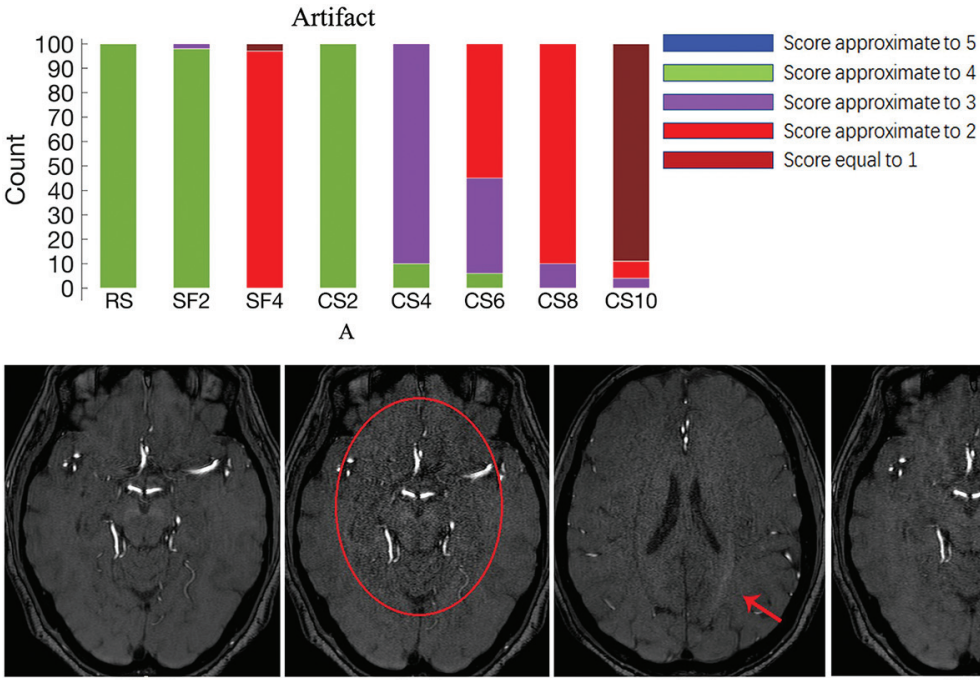

C(SF2)

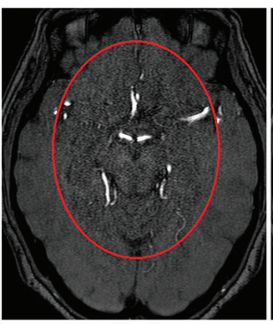

$\mathrm{D}$ (SF4)

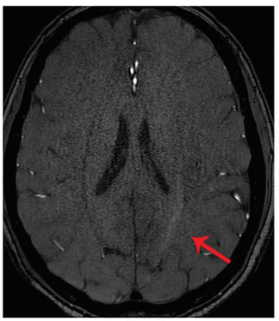

$\mathrm{E}(\mathrm{SF} 4)$

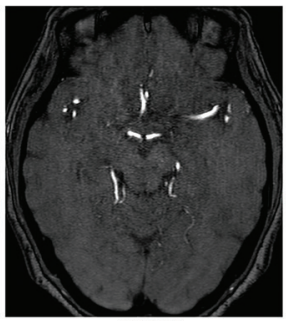

$\mathrm{F}(\mathrm{CS} 6)$

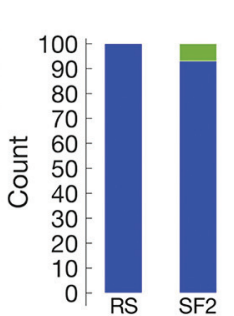

Overall image quality
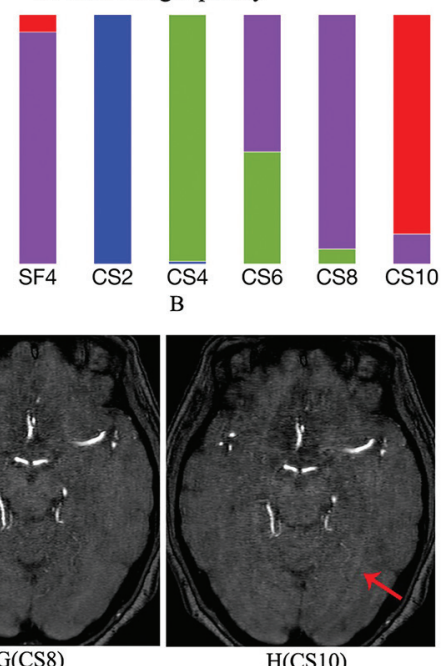

FIG 3. Subject count of scores for the 8 protocols about the artifacts $(A)$ and overall image quality $(B)$ and transverse images of different protocols from 1 healthy subject $(C-H)$. Speckled noise is found in the center region of SF4 image (red circle, D) compared with SF2 (C), and the SENSE artifact is observed in SF4 (red arrow, E, different section from D). Curved striped pattern artifacts are obvious for CS8 (G) and CS10 (H) (red arrows), but negligible for CS6 (F).

the center region of images with SF4 (Fig 3D, -E). Curved striped pattern artifacts were obvious for CS8 and CS10 (Fig 3G, -H). For scores of artifacts and overall image quality, no significant differences were found in pair-wise comparisons among RS, SF2, and CS2 (Online Supplemental Data), and the mean scores decreased gradually as the SENSE or CS-SENSE acceleration factor increased (Fig 3).

Diagnostic Confidence for Arterial Aneurysm and Stenosis. No significant difference in the diagnostic confidence was found in the pair-wise comparisons among RS, SF2, and CS2 for either arterial stenosis or an aneurysm $(P>.05$, Fig $4 A,-B)$. All arterial aneurysms (with diameters ranging from approximately 3 to $8 \mathrm{~mm}$ and positions listed in Online Supplemental Data) and most cases of arterial stenosis were well-diagnosed with RS, SF2, and CS2 (nearly all scores were equal to 3 and were considered acceptable), diagnosed with relative certainty with CS4 and CS6 (most scores were equal to 2 and the others were equal to 3 , acceptable), and unclearly diagnosed with SF4, CS8, and CS10 (most the scores were $<2$ ). Notably, the stenoses in the large arteries (listed in the Online Supplemental Data) could be successfully diagnosed on images of all 8 sequences. The zoomed MIP images obtained by the 8 sequences for 1 patient with mild stenosis in the BA (Fig 4C, $-J$ ) and for another patient with a tiny arterial aneurysm of the right ICA (Fig $4 K,-R$ ) are shown.

\section{DISCUSSION}

In this study, the CS-SENSE technique with 5 different acceleration factors was evaluated for brain 3D TOF-MRA in a cohort of 100 patients with images collected from multiple centers. A reference sequence without acceleration and 2 sequences using the conventional SENSE technique were included for comparison. CS-SENSE with an acceleration factor of 4 could provide balanced image quality and acquisition times.
Obviously reduced image information was found from the quantitative measurements (tendency of RSIs or SSIM) when the CS-SENSE acceleration factors gradually increased from 2 to 10 . The possible reason was that sparser data sampling was achieved for scans with higher acceleration factors. ${ }^{13}$ Although the mean $C R_{\text {artery/wm }}$ and $C R_{\text {artery/csf }}$ values slowly decreased as the CSSENSE factor increased, no significant difference was found between CRs for any pairs of SF2, CS2, and CS4, which indicated that imaging contrasts (between assessed arteries and WM/CSF) of CS4 were comparable with those of CS2 and SF2, despite the increased acceleration factor.

CS2 could generate images with almost equally acceptable quality compared with RS but with a reduction of $47.95 \%$ in scan time, considering both the comparisons of the qualitative and quantitative assessments. As the CS-SENSE acceleration factor increased from 2 to 4 though there were obvious decreases in some quantitative and qualitative assessments, images of both CS2 and CS4 achieved acceptable image quality through qualitative evaluations, including the visualization of all arteries, qualitative scores of artifacts and overall image quality, and diagnostic confidence of stenosis or aneurysm, according to the criteria in this study. In this sense, CS4 yielded image quality lower than that of CS2, which was a natural outcome of exaggerated undersampling, but CS4 images were still acceptable for diagnosis from all the perspectives under evaluation in our study.

Given the reduced scan time (reduced by $48.68 \%$ ) compared with CS2, CS4 could be a practical setup in routine clinical scanning. As the CS-SENSE acceleration factor increased from 4 to 6 , visualization of the large and medium arteries was still acceptable, agreeing with the previous study by Yamamoto et al. ${ }^{12}$ However, visualization of 2 small arteries (LAChA and RAChA) became unacceptable according to the criteria in this study, which might render CS6 unpromising for examinations of some small arterial disorders, such as the evaluation of small intracranial 


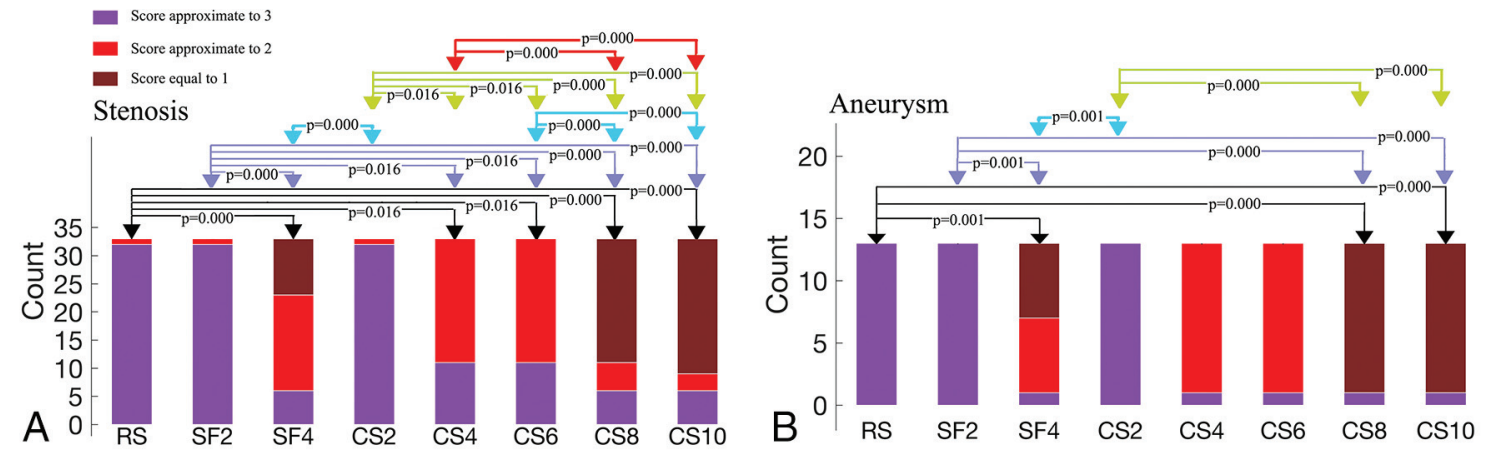

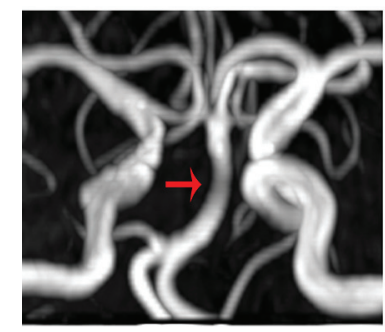

C(RS)

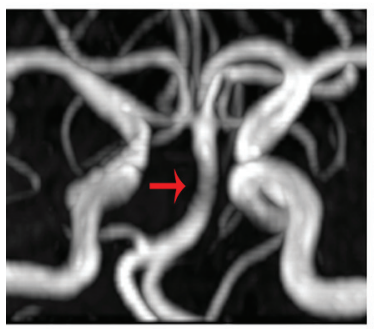

G(CS4)

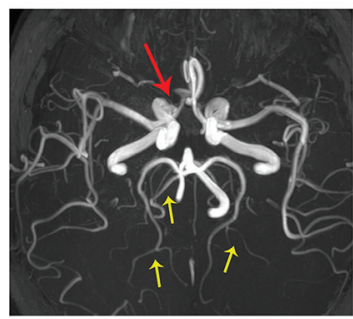

K(RS)

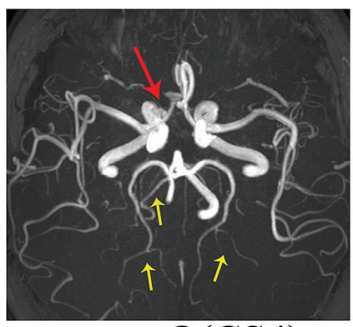

$\mathrm{O}(\mathrm{CS} 4)$

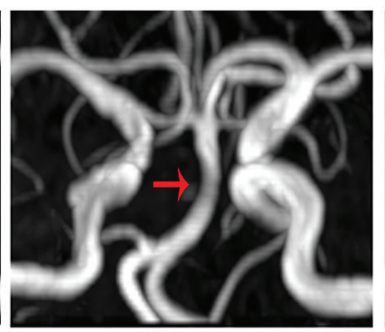

D(SF2)

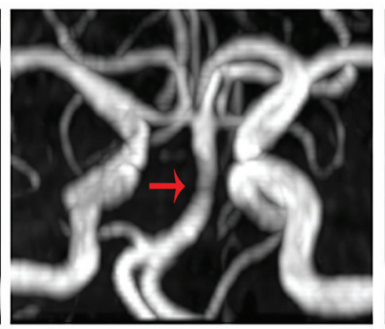

$\mathrm{H}(\mathrm{CS} 6)$

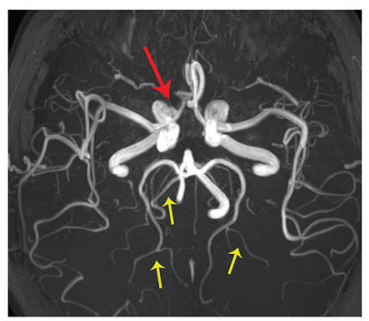

L(SF2)

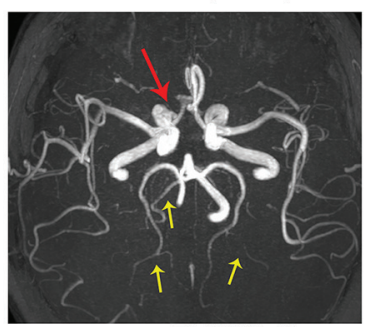

P(CS6)

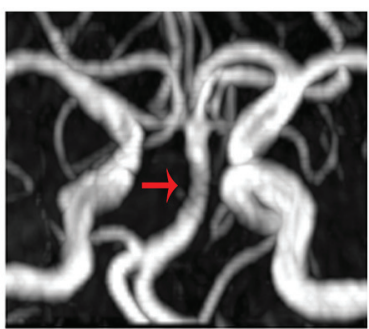

$\mathrm{E}(\mathrm{SF} 4)$

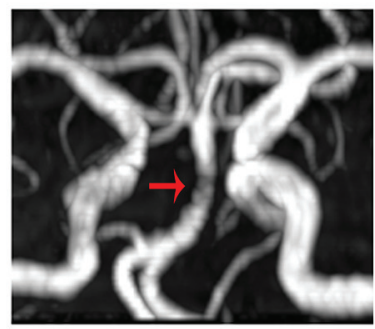

I(CS8)

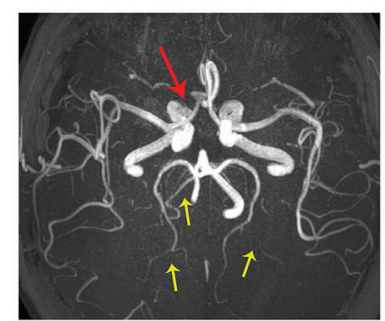

M(SF4)

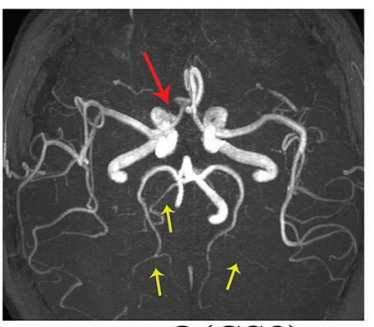

$\mathrm{Q}(\mathrm{CS} 8)$

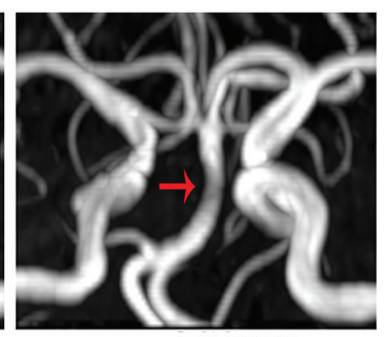

$\mathrm{F}(\mathrm{CS} 2)$

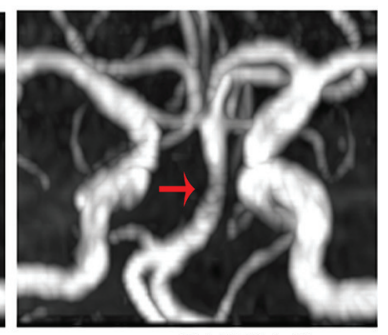

$\mathrm{J}(\mathrm{CS} 10)$

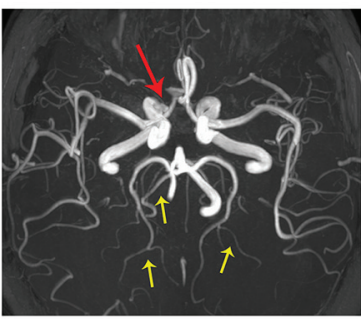

$\mathrm{N}(\mathrm{CS} 2)$

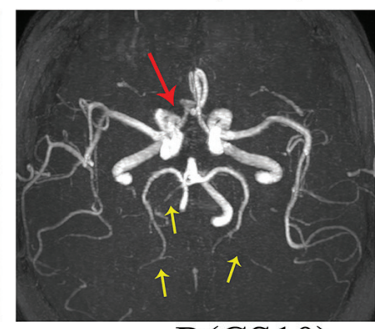

$\mathrm{R}(\mathrm{CS} 10)$

FIG 4. Patient count of scores for the 8 protocols of the arterial stenosis $(A)$ and aneurysms $(B)$. Groups with significant differences $(P<.05)$ are connected by double arrow lines. Zoomed MIP images obtained by the 8 sequences for 1 patient with mild stenosis (anterior view). The BA has a smooth boundary and uniform signal distribution in the region of the stenosis (red arrow) in images of RS (C), SF2 (D), and CS2 (F); it is visually acceptable in images of CS4 $(G)$ and CS6 $(H)$, but with rough boundaries and granulated signal distributions in images of SF4 (E), CS8 (I), and CS10 (). Zoomed MIP images obtained by the 8 sequences for a patient with a tiny arterial aneurysm in the eye segment of right ICA (inferior view). The arterial aneurysm has an explicit boundary (red arrow) and can be well-diagnosed in RS (K), SF2 (L), CS2 (N), CS4 (O), and CS6 (P) (mean score $=3$ ) and can be diagnosed with relative certainty in CS8 (Q) and SF4 (M) (mean score = 2); but the boundary becomes rough and unclear in CS10 $(R)$. Additionally, 3 distal arterial branches were marked by yellow arrows in each image $(K-R)$. These arterial branches are clear and continuous in RS $(K)$, SF2 (L), CS2 (N), CS4 (O), and CS6 (P), but become discontinuous or even invisible in SF4 $(M)$, CS8 $(Q)$, and CS10 (R). 
vasculature and arteriovenous malformations in challenging regions of the skull base.

As the acceleration factor increased to 8 or 10, visualization of small and medium arteries became unacceptable and the diagnostic confidence of arterial stenosis and aneurysms also became less convincing (the small size of aneurysms in this study and excessive sparsity of CS-SENSE might be possible reasons for the misdiagnosis of arterial aneurysms in large arteries). Moreover, curved striped pattern artifacts became obvious, the boundaries of the arteries became rougher, and some small arteries became discontinuous or missing in images. All these factors increase the potential risk of misdiagnosis or unclear diagnosis of possible pathologies. Nevertheless, reliable visualization for large arteries and a high diagnostic rate for all 5 stenoses in large arteries could be achieved by CS10. Considering the unprecedented short scan time (48 seconds), CS10 might be a fast approach for visualization or stenosis screening for large arteries (eg, ICA occlusion for emergency intravascular intervention).

Although conventional SENSE-accelerated TOF-MRA (such as SF2) is clinically effective and widely used for the noninvasive evaluation of cerebrovascular pathology, the long scan duration and vulnerability to motion artifacts can still be problematic for its clinical applications. ${ }^{25,26}$ From both qualitative assessments and quantitative measurements, no significant difference was found between CS2 and SF2. The RSIs of assessed arteries, WM, and CSF in images in CS4 were obviously lower than those in SF2 due to the sparse sampling for CS-SENSE; however, the CRs were basically equivalent. Furthermore, considering the acceptable visualization of assessed arteries, overall image quality, relatively certain diagnostic confidence of arterial stenosis and aneurysm, and reduced scan time (reduced by $46.33 \%$ compared with SF2), CS4 could be considered a better choice than SF2 in routine clinical settings. Additionally, the overall performance of CS4 was much better than that of SF4. Even for CS6, the RSIs for all assessed arteries, the mean scores for most arteries (14/17, 82\%) and the mean scores of artifacts (2.51 versus 1.97), and the overall image quality (3.45 versus 2.93) were still higher than those with SF4. Moreover, the diagnostic confidence for arterial aneurysms and stenosis with CS6 was higher than that with SF4. These observations suggest the use of higher acceleration factors with CSSENSE in TOF-MRA clinical setups than with conventional SENSE, which was consistent with previous studies. ${ }^{8,12}$

Speckled noise and SENSE artifacts were commonly observed in the central part of TOF-MRA images with SF4, which was also mentioned in previous studies, ${ }^{8}$ while these SENSE ghost artifacts were rarely seen on the CS-SENSE images. However, in this study, curved striped pattern artifacts were observed when the CS-SENSE acceleration factor was $>6$ (obvious in CS8 and CS10), especially around the skull boundaries. The possible origination of these curved striped pattern artifacts was thought to be related to the phase-encoding direction and sparse sampling, possibly derived from the outer edge of the skull. ${ }^{18}$

The current study has several limitations. First, the final diagnosis of the arterial pathologies was confirmed by comprehensive consideration of the MRA images, the routine MR images (T1, T2, FLAIR, DWI, and SWI), and any other available clinical data. DSA was not considered essential in this study due to its invasive procedure. Furthermore, several studies have verified a good correlation between MRA and DSA., ${ }^{4,5,27}$ Second, only 2 cerebrovascular pathologies (arterial stenosis and aneurysm) were assessed in this study. The performance of MRA with different CS-SENSE acceleration factors for other possible vessel pathologies (eg, vascular malformation) still merits further investigation. Additionally, the influence of CS-SENSE on the diameters of aneurysms or rates of stenosis was not included in the study, which would also be considered in future studies. Third, no comparison was performed among different centers in this study. Fourth, the number of iterations in the CS reconstruction that might have influenced the image quality ${ }^{6}$ was not included. Fifth, only 1 neuroradiologist was chosen for ROI drawing. Although we believe that the neuroradiologist with $>10$ years of experience was professional in drawing ROIs, $\geq 2$ readers would be better. Sixth, spiral TOF-MRA, which potentially delivers high-quality intracranial vessel imaging at a short scan time, was not included for comparison in the study ${ }^{28,29}$ and will be studied in future work.

\section{CONCLUSIONS}

The CS-SENSE technique with an acceleration factor of 4 is generally acceptable for brain 3D TOF-MRA in clinical setups at 3T with balanced image quality and acquisition times (117 seconds), while CS6 (79 seconds) can be used for examinations in which small arteries are not evaluated and CS10 (48 seconds) may be suitable for fast visualization of large arteries.

\section{ACKNOWLEDGMENTS}

We thank Rongkai Ju and Yishi Wang for support in writing this article.

Disclosures: Jiazheng Wang-UNRELATED: Employment: Philips Healthcare. Liangjie Lin—UNRELATED: Employment: Philips Healthcare.

\section{REFERENCES}

1. Chung MS, Jung SC, Kim SO, et al. Intracranial artery steno-occlusion: diagnosis by using two-dimensional spatially selective radiofrequency excitation pulse MR imaging. Radiology 2017;284:834-43 CrossRef Medline

2. Mandell DM, Mossa-Basha M, Qiao Y, et al. Vessel Wall Imaging Study Group of the American Society of Neuroradiology. Intracranial Vessel Wall MRI: Principles and Expert Consensus Recommendations of the American Society of Neuroradiology. AJNR Am J Neuroradiol 2017;38:218-29 CrossRef Medline

3. Park JE, Jung SC, Lee SH, et al. Comparison of 3D magnetic resonance imaging and digital subtraction angiography for intracranial artery stenosis. Eur Radiol 2017;27:4737-46 CrossRef Medline

4. Debrey SM, Yu H, Lynch JK, et al. Diagnostic accuracy of magnetic resonance angiography for internal carotid artery disease: a systematic review and meta-analysis. Stroke 2008;39:2237-48 CrossRef Medline

5. van Rooij WJ, Sprengers ME, de Gast AN, et al. 3D rotational angiography: the new gold standard in the detection of additional intracranial aneurysms. AJNR Am J Neuroradiol 2008;29:976-79 CrossRef Medline

6. Fushimi Y, Fujimoto K, Okada T, et al. Compressed sensing 3dimensional time-of-flight magnetic resonance angiography for cerebral aneurysms: optimization and evaluation. Invest Radiol 2016;51:228-35 CrossRef Medline 
7. Li B, Li H, Dong L, et al. Fast carotid artery MR angiography with compressed sensing based three-dimensional time-of-flight sequence. Magn Reson Imaging 2017;43:129-35 CrossRef Medline

8. Lin Z, Zhang X, Guo L, et al. Clinical feasibility study of 3D intracranial magnetic resonance angiography using compressed sensing. J Magn Reson Imaging 2019;50:1843-51 CrossRef Medline

9. Bhagat YA, Emery DJ, Naik S, et al. Comparison of generalized autocalibrating partially parallel acquisitions and modified sensitivity encoding for diffusion tensor imaging. AJNR Am J Neuroradiol 2007;28:29398 Medline

10. Preibisch C, Wallenhorst T, Heidemann R, et al. Comparison of parallel acquisition techniques generalized autocalibrating partially parallel acquisitions (GRAPPA) and modified sensitivity encoding (mSENSE) in functional MRI (fMRI) at 3T. J Magn Reson Imaging 2008;27:590-98 CrossRef Medline

11. Larkman DJ, Nunes RG. Parallel magnetic resonance imaging. Phys Med Biol 2007;52:R15-55 CrossRef Medline

12. Yamamoto T, Fujimoto K, Okada T, et al. Time-of-flight magnetic resonance angiography with sparse undersampling and iterative reconstruction: comparison with conventional parallel imaging for accelerated imaging. Invest Radiol 2016;51:372-78 CrossRef Medline

13. Lustig M, Donoho D, Pauly JM. Sparse MRI: the application of compressed sensing for rapid MR imaging. Magn Reson Med 2007;58:1182-95 CrossRef Medline

14. Lu SS, Qi M, Zhang X, et al. Clinical evaluation of highly accelerated compressed sensing time-of-flight MR angiography for intracranial arterial stenosis. AJNR Am J Neuroradiol 2018;39:1833-38 CrossRef Medline

15. Greve T, Sollmann N, Hock A, et al. Highly accelerated time-offlight magnetic resonance angiography using spiral imaging improves conspicuity of intracranial arterial branches while reducing scan time. Eur Radiol 2020;30:855-65 CrossRef Medline

16. Zhang X, Cao YZ, Mu XH, et al. Highly accelerated compressed sensing time-of-flight magnetic resonance angiography may be reliable for diagnosing head and neck arterial steno-occlusive disease: a comparative study with digital subtraction angiography. Eur Radiol 2020;30:3059-65 CrossRef Medline

17. Suh $\mathrm{CH}$, Jung SC, Lee $\mathrm{HB}$, et al. High-resolution magnetic resonance imaging using compressed sensing for intracranial and extracranial arteries: comparison with conventional parallel imaging. Korean J Radiol 2019;20:487-97 CrossRef Medline
18. Yamamoto T, Okada T, Fushimi $\mathrm{Y}$, et al. Magnetic resonance angiography with compressed sensing: an evaluation of Moyamoya disease. PLoS One 2018;13:e0189493 CrossRef Medline

19. Geerts-Ossevoort L, de Weerdt E, Duijndam A, et al. Compressed SENSE. Speed done right. Every time. Philips Healthcare, Netherlands, 2018. https://philipsproductcontent.blob.core.windows.net/assets/ 20180109/619119731f2a42c4acd4a863008a46c7.pdf. Accessed May 1, 2020

20. Bratke G, Rau R, Weiss K, et al. Accelerated MRI of the lumbar spine using compressed sensing: quality and efficiency. J Magn Reson Imaging 2019;49:e164-75 CrossRef Medline

21. Lustig M, Pauly JM. SPIRiT: Iterative self-consistent parallel imaging reconstruction from arbitrary k-space. Magn Reson Med 2010;64:45771 CrossRef Medline

22. Okubo G, Okada T, Yamamoto A, et al. MP2RAGE for deep gray matter measurement of the brain: a comparative study with MPRAGE. $J$ Magn Reson Imaging 2016;43:55-62 CrossRef Medline

23. Altahawi FF, Blount KJ, Morley NP, et al. Comparing an accelerated 3D fast spin-echo sequence (CS-SPACE) for knee 3-T magnetic resonance imaging with traditional 3D fast spin-echo (SPACE) and routine 2D sequences. Skeletal Radiol 2017;46:7-15 CrossRef Medline

24. Wang Z, Bovik AC, Sheikh HR, et al. Image quality assessment: from error visibility to structural similarity. IEEE Trans Image Process 2004;13:600-12 CrossRef Medline

25. Willinsky RA, Taylor SM, TerBrugge K, et al. Neurologic complications of cerebral angiography: prospective analysis of 2,899 procedures and review of the literature. Radiology 2003;227:522-28 CrossRef Medline

26. Wu H, Block WF, Turski PA, et al. Noncontrast-enhanced threedimensional (3D) intracranial MR angiography using pseudocontinuous arterial spin labeling and accelerated 3D radial acquisition. Magn Reson Med 2013;69:708-15 CrossRef Medline

27. Borisch I, Horn M, Butz B, et al. Preoperative evaluation of carotid artery stenosis: comparison of contrast-enhanced MR angiography and duplex sonography with digital subtraction angiography. AJNR Am J Neuroradiol 2003;24:1117-22 Medline

28. Sartoretti T, van Smoorenburg L, Sartoretti E, et al. Ultrafast intracranial vessel imaging with non-Cartesian spiral 3-dimensional time-of-flight magnetic resonance angiography at $1.5 \mathrm{~T}$. Invest Radiol 2020;55:293-303 CrossRef Medline

29. Sartoretti T, Sartoretti E, Schwenk A, et al. Clinical feasibility of ultrafast intracranial vessel imaging with non-Cartesian spiral 3D time-of-flight MR angiography at 1.5T: an intra-individual comparison study. PLoS One 2020;15:e0232372 CrossRef Medline 